\title{
Comparison of Therapeutic Results of Using Short and Long Proximal Femoral Nail (PFN) in Patients with Intertrochanteric Fracture
}

\section{Payam Mohammad Hoseini*, Mohammad Fakoor, Mohammad Ali Ghasemi, Seyed Mohammad Mohammadi}

Department of Orthopaedics and Traumatology, Ahvaz Jundishapur University of Medical Sciences, Ahvaz, Iran

Study Area: Ahvaz, Iran

Coordinates: $31^{\circ} 19^{\prime} 13^{\prime \prime N}$; $48^{\circ} 40^{\prime} 09^{\prime \prime E}$

Key words: Limb length discrepancy, Harris Hip Score, Post-surgical complications

This study was approved at the Ethics Committee of the Jundishapur University of Ahvaz (Code: IR.AJUMS.REC. 1397.479).

\section{Introduction:}

Fractures around the hip joint are a part of the larger group of pelvic cases that are common in the elderly and ratio is increasing day by day (Horowitz , 1966; Rockwood et al., 2010; Loizou et al., 2010). The underlying cause of intertrochanteric fracture is the combination of increased bone fragility and reduction of muscle tonicity of the related region (Min et al., 2007). The main treatment of such fractures is only the surgical procedure, however, nonsurgical treatments also have an acceptable level of recovery, but currently, such treatments is not in use because of the need for long-term immobilization (Min et al., 2007; Ramakrishnan et al., 2004). For intertrochanteric fractures, various surgical treatments such as Dynamic Hip Screw (DHS), Intramedullary Nailing (IMN), Proximal Femoral Nail (PFN), bipolar hemiarthroplasty, and External Fixator (EF) have been introduced but each of them have their own advantages and disadvantages (Horowitz , 1966; Rockwood et al., 2010; Seyfettinoglu et al., 2010; Bhandari et al., 2003; Soucanye et al., 2012; Schmidt \& Swiontkowski, 2002). Despite the good results of DHS, sometimes even with all the required precautions, the

\section{Abstract}

An attempt was made to compare the treatment outcomes, the complications during and after surgery, and the level of satisfaction of the patients treated either with short or long Proximal Femoral Nail (PFN). Total of 40 patients aged 50 to 70 years with intertrochanteric fracture (Type $\mathrm{A} 2$ and 31-A1/ OTA), underwent surgery with short or long PFN method were considered under this retrospective study. Duration of surgery, the amount of bleeding during surgery, rate of union, Harris Hip Score, post-surgical complications including surgical failure, deep infection, limb length discrepancy, the status of embolization of patients and the status of walking with or without auxiliary devices were evaluated and compared between two groups. The duration of surgery in the short PFN group was significantly lower than the long PFN group. But, there was no statistically significant difference in any parameter between the two groups was evidenced. According to the HHS classification, the mean score of HHS obtained from both groups was placed in the "good" level. Finally, as per our results, both the methods could be suggested as a convenient and useful method for treating intertrochanteric fractures.

results are not as per the expectation (Rha et al., 1993; Christodoulou \& Sdrenias, 200o). Some researchers suggested that IM Rod input lower the pressure to the site of the fracture with less chance of displacement, therefore, the chance of collapse of the neck and the duration of operation and bleeding during surgery also would be less. The most famous instruments used since the early 1980 s are Gamma Nail, Trochanteric Antegrade Nail, and PFN. The general principles of using these devices are the same and using these devices is less likely to bleed and damage to large trochanter and muscle (Harandi et al., 2006). Although the difference in therapeutic results from short and long PFNs is not explicitly stated, some studies have highlighted the cost of using long PFN, the surgical time, the amount of bleeding during surgery and its complications more than the short PFN. Also, the results of studies have shown that the use of long PFN does not reduce the chances of Periprosthetic fracture and reoperation (Dunn et al., 2016; Kanakaris et al., 2015; Boone et al., 2014; Kleweno et al., 2014). In contrast to some studies, there was no significant difference between the clinical outcomes of the two methods, and both methods 
were useful in treating intertrochanteric fractures (Kleweno et al., 2014; Vaughn et al., 2015; Okcu et al., 2013). There is still controversy about the magnitude of postoperative complications such as surgical fixation failure, the need for hospitalization, and mortality rates associated with the use of short or long PFN. Our study aimed to compare the treatment outcomes, perioperative complications, and the level of satisfaction of patients treated with either short or long PFN.

\section{Methodology:}

In the year 2016-2017, this study was conducted as a retrospective study on 40 patients aged 50 to 70 years with intertrochanteric fracture (Type $\mathrm{A}_{2}$ and 31-A1/ OTA) referred to Imam Khomeini and Golestan hospitals of Ahvaz that underwent surgery with short $(n=20)$ or long $(n=20)$ PFN method. The length of the nail used in the short PFN group was between 20 and $24 \mathrm{~cm}$ and in the long PFN group was between 36 and $40 \mathrm{~cm}$ (Fig.-1a,b). After collecting related information from archival files of the hospital, including demographics (age at surgery and gender) and clinical (duration of surgery and the amount of bleeding during surgery), the qualified patients were contacted to attend the clinic. Proper consents were taken from the patients underwent clinical examinations including the lameness and limb shortening under the supervision of an orthopedic specialist. All patients were evaluated with radiographic assessment, and the previous graphs related to one year after surgery and also taken during the follow-up period were also evaluated. Non-union was considered if there was not complete union within a period of 6 months after surgery was noticed. Harris Hip Score questionnaire (HHS) was also used to evaluate and compare the functional recovery of patients in the two groups. Postsurgical complications, including surgical failure (Patients for whom surgical revision was performed as a surgical failure, and the cause of revision was also recorded), deep infection (based on the amount of ESR, CRP in the lab tests during follow-up, limb length discrepancy (LLD, the status of embolization of patients and the status of walking with or without auxiliary devices were also taken under consideration. According to the classif ication of Harris Hip Score, the score was considered as "Excellent" (100-90), "Good" (89-80), "Fair" (79-70), and "Poor" (less than 70). The amount of bleeding during surgery was calculated according to the number of blood gases and in a milliliter. The surgical duration was considered as a skin-to-skin period in a minute. Inclusion criteria were patients aged 50 to 70 years, passed at least one year of surgery, patients with intertrochanteric fractures type $\mathrm{A} 1$ and $\mathrm{A} 2$ according to $\mathrm{AO}$ classification. Exclusion criteria were patients with a history of fractures in the lower limbs, multiple trauma patients, patients with diabetes, patients with coagulation problems and addiction to opioids. All the data were compared between the two groups and the results of the

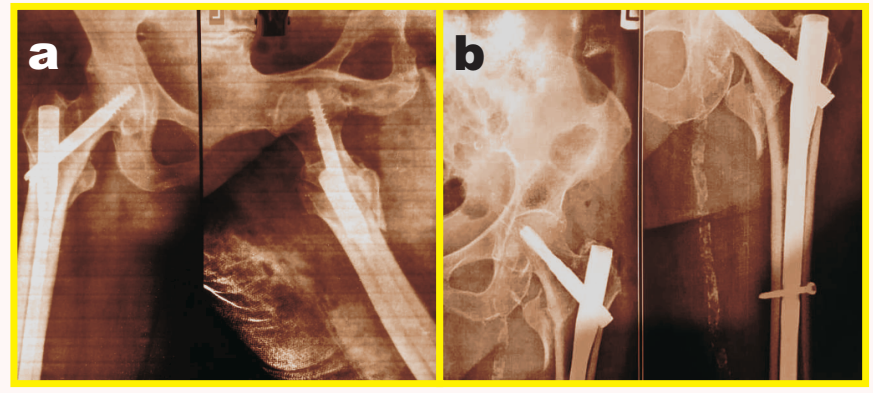

Figure 1. A sample of radiographic assessment 6 month after surgery for long PFN (a) and short PNF (b)

treatment were evaluated and compared with statistical tests. Independent t-test and Chi-square test were used to compare quantitative and qualitative, respectively. Data were analyzed using SPSS 22 software and the signif icance level was considered as $\mathrm{p}$-value $<0.05$.

\section{Results:}

There was no significant difference between the mean age of the patients in both groups. In both groups, the number of females was higher than males, but the two groups show the same trend in the sexual distribution of patients and there was no significant difference between them. The number of patients with fracture type 31-A2 OTA was more than $31-A 1$ OTA in both groups, but in terms of fracture type, there was no significant difference between the two groups. The prevalent of fracture in the left side was more than the right side in both groups, but even than there was no significant difference between the distribution of patients based on the fracture side in both groups (Table-1).

Table-1: Demographic and clinical information short Vs long PFN groups

\begin{tabular}{lllll}
\hline Variable & & Short PFN & Long PFN & P-value \\
\hline Age (year) & (Mean \pm SD) & $64.07 \pm 5.62$ & $63.18 \pm 5.12$ & $>0.96$ \\
Gender & Women & $12(60 \%)$ & $11(55 \%)$ & $>0.92$ \\
$(\mathrm{~N}, \%)$ & Men & $8(40 \%)$ & $9(45 \%)$ & \\
Fracture type & OTA $31-A 1$ & $5(25 \%)$ & $6(30 \%)$ & $>0.92$ \\
$(\mathrm{~N}, \%)$ & OTA $_{31-A 2}$ & $15(75 \%)$ & $14(70 \%)$ & \\
Location & Right & $6(30 \%)$ & $8(40 \%)$ & $>0.92$ \\
$(\mathrm{~N}, \%)$ & Left & $14(70 \%)$ & $12(60 \%)$ & \\
\hline
\end{tabular}

Although the amount of bleeding during surgery in the short PFN group was lower, there was no statistically significant difference was evidenced between the two groups. The duration of surgery in the short PFN group was significantly lower than the long PFN group $(\mathrm{p}<0.0001)$. The mean duration of the union in the long PFN group was shorter than the short PFN group, however, the difference was not found to be statistically significant between these two groups. Delay union rate was found in three patients in the short PFN group (15\%) and in 2 patients in the long PFN group $(10 \%)$. The difference in the incidence of this complication was not found to be statistically significant between these two groups. One patient in the short PFN group (5\%), and 3 patients in the long PFN group $(15 \%)$ 
ORIGINAL ARTICLE

Table-1: Demographic and clinical information short Vs long PFN groups

\begin{tabular}{|c|c|c|c|c|}
\hline \multicolumn{2}{|c|}{ Variable } & Short PFN & Long PFN & P-value \\
\hline \multicolumn{2}{|c|}{ Blood loss (ml) $(\mathrm{M} \pm \mathrm{SD})$} & $71.22 \pm 24.17$ & $81.63 \pm 26.77$ & $>0.071$ \\
\hline \multicolumn{2}{|c|}{ Operative time $(\mathrm{min})(\mathrm{M} \pm \mathrm{SD})$} & $54.07 \pm 9.78$ & $81.34 \pm 11.43$ & $<0.0001$ \\
\hline \multicolumn{2}{|c|}{ Union time (month) $(\mathrm{M} \pm \mathrm{SD})$} & $9.01 \pm 2.72$ & $8.14 \pm 2.47$ & $>0.28$ \\
\hline \multicolumn{2}{|c|}{ Delay Union (N, \%) } & $3(15 \%)$ & $2(10 \%)$ & $>0.092$ \\
\hline \multicolumn{2}{|c|}{ Malunion (N, \%) } & $1(5 \%)$ & $3(15 \%)$ & $>0.088$ \\
\hline \multicolumn{2}{|c|}{ Nonunion (N, \%) } & $3(15 \%)$ & $1(5 \%)$ & $>0.088$ \\
\hline \multirow[t]{5}{*}{ HHS } & Excellent (N, \%) & $7(35 \%)$ & $5(25 \%)$ & $>0.16$ \\
\hline & Good (N, \%) & $11(55 \%)$ & $10(50 \%)$ & \\
\hline & Fair (N, \%) & $1(5 \%)$ & $3(15 \%)$ & \\
\hline & Poor $(\mathrm{N}, \%)$ & $1(5 \%)$ & $2(10 \%)$ & \\
\hline & Mean \pm SD & 87.56 (Good) & $81.22($ Good $)$ & $>0.22$ \\
\hline \multicolumn{5}{|c|}{ Complication (N, \%) } \\
\hline & Deep Infection & $2(10 \%)$ & $1(5 \%)$ & $>0.08$ \\
\hline & LLD & $1(5 \%)$ & o $(0 \%)$ & $>0.091$ \\
\hline \multicolumn{5}{|c|}{ Ambulatory Status (N, \%) } \\
\hline & Independent & $13(65 \%)$ & $16(80 \%)$ & $>0.085$ \\
\hline & with walking aid & $5(25 \%)$ & $3(15 \%)$ & \\
\hline & Non- ambulator & $2(10 \%)$ & $1(5 \%)$ & \\
\hline & Decaesed & o (o\%) & o $(0 \%)$ & \\
\hline Hosp & stay (Day) (M+SD) & $5.1 \pm 2.97$ & $4.8 \pm 2.78$ & $>0.72$ \\
\hline
\end{tabular}

were suffered from malunion, but the difference in the incidence of this complication between the two groups was not statistically significant. Three patients in the short PFN group (15\%), and 1 patient in the long PFN group (5\%) experienced failure in the union. Although the rate of nonunion was reported more frequently in the patients with short PFN group, the difference in complication was not statistically significant between these two groups. In the group treated with long PFN, the number of patients in the level of excellent and good was superior to short PFN group, but there was no significant difference between the distribution of patients based on the classification def ined by the HHS scores. According to the HHS classification, the mean HHS obtained from both groups was placed in the "good" level. Although the mean of this score was superior in the long PFN group, there was no significant difference between these two groups. Two patients in the short PFN group (10\%) and 1 in the long PFN group (5\%) reported deep infection that incidence of this complication was also not found to differ statistically between the two groups. For a patient in the short PFN group, infection led to a surgical revision and other patients improved with medication therapy. The rate of surgical failure in the short PFN group (20\%) was significantly higher than the long PFN group (10\%). All 6 patients were underwent surgical revision. The reason for the revision in the short PFN group was 1 case of deep infection, 1 case of non-union and 2 cases of refracture. The cause of revision in the long PFN group was 1 case of non-union and 1 case of fall and re-fracture, which was based on the causes and considering that 3 patients had failed due to re-fracture. Surgical failure in the two groups due to the low number of cases have not been compared. Only one patient in the short PFN group had LLD less than 2 $\mathrm{cm}$, which was not symptomatic and treated with Heel Lift. Although patients in long PFN group were more ambulatory and less dependent on the use of auxiliary devices, there was no significant difference between these two groups. No mortality in one year span after surgery was seen for both the groups. Although patients in the short PFN group had a longer stay in the hospital, there was no signif icant difference between these two groups in terms of hospitalization time (Table-2).

\section{Discussion:}

Our study was aimed to compare the therapeutic outcomes of using short and long PFN in treatment of intertrochanteric fracture. Result evidenced for the amount of bleeding during surgery in the short PFN group was less, but statistically no difference. But, Dunn et al. (2016), reported the amount of received blood in the short nail group was $8.8 \%$ (39 ml) which was lower than that of the long nail group and thus both the groups were seen to be statistically differentiated from each other.

We found the duration of surgery in the short PFN group was significantly lower than the PFN group which supports the result of Dunn et al. (2016). In our study, the mean duration of the union in the long PFN group was shorter than the short PFN group, but the differences were not statistically significant. The differences in incidence complications including delayed union, malunion and nonunion in two groups were not statistically significant. More or less Dunn et al. (2016)'s results were consistent with ourstudy in this regard.

In the study of Okcu et al. (2013), the union rate was reported $100 \%$ in both groups and it was more than the reported rate of our study. There was no significant difference between the distribution of patients according to the classification defined in the HHS criteria. The mean functional score for both groups was categorized as "good" according to the classification of HHS and there was no significant difference in the mean functional score between the two groups. Further, Okku et al. (2013), also reported more or less the same result in this regard. In the present study, the patients in both groups were had better functional scores than Okcu et al. (2013), which may be due to the higher age of patients and relationship between functional score and age of patients (67 to 95 years) in their study as compared to the present one.

There was no significant difference between the two groups regarding the incidence of deep infection was evidenced. In a study by Okcu et al. (2013), only one patient $(6.7 \%)$ had a deep infection in the short PFN group, which was less than the observed rate in the study.

There was no significant difference in the rate of surgical failure due to complications in both groups. In the study of Okcu et al. (2013), only two patients (13.33\%) in the 
short PFN group had undergone a surgical revision due to deep infection, and the rate of surgical revision in their study was less than the present study, but the similarity of the two studies was further incremented in the number of revision in the short PFN group.

In the study of Kleweno et al. (2014) a comparison was made on the outcomes of short and long CMN methods, 7 patients (3.2\%) in the CMN group and 12 patients (3.5\%) in the long $\mathrm{CMN}$ group reported surgical failure and major revision and nail reduction were performed for them. The reasons for the revision were AVN (avascular necrosis), femoral head, advanced arthritis, screw breakage, and LLD. The rate of surgical revision in the Kleweno et al. (2014)'s study was lower than that the present study, but the similarity of two studies was a higher number of revisions in the short nail group. In the study of Dunn et al. (2016), the rate of surgical revision in the short $\mathrm{CMN}$ group was $5 \%$ and in the long CMN group was $3.8 \%$, which did not differ signif icantly. The rate of surgical revision in the Dunn et al. (2016)'s study was lower than that observed in the present study, but the similarity of the two studies was the higher number of revision in the short nail group. In the study of Vaughn et al. (2015), surgical failure in the short CMN group was $5 \%$ and in the CMN group was $6.2 \%$ (statistically no difference), which was less than the reported failure rate in the present study, but similarity of two studies was a higher number of surgical failure in the short nail group. In the study of Krigbaum et al. (2016), the failure rate within 1 year after surgery was not significantly different in both long and short CMN groups.

In our study, none of the patients in either group had died of death but Okcu et al. (2013), reported the mortality rate in the short PFN group as $17 \%$ and in the long PFN group as $18 \%$, but it did not show any statistical differences. The mortality rate in the study of Okcu et al. (2013) was greater than the present study, which may be due to the older age group of patients (67 to 95 years) in their study. Hospitalization time for our study and Krigbaum et al. (2016) both were not found to differ statistically between the two long and short CMN groups.

Conclusively our results evidenced that the two short and long PFN treatment methods did not significantly differ in terms of functional improvement, the incidence of complications, and hospitalization duration, and both methods could be used as a convenient and useful method for treating intertrochanteric fractures. However, due to complications during surgery, including surgical bleeding and short duration of operation in the short PFN group, this method can be fruitful in patients with higher anesthetic risk or with more coagulation disorders and also due to the shorter PFN is more economical where this is important, this approach can be favored by the long PFN.

\section{Acknowledgment:}

We are thankful to all the particinats for their kind cooperation during the study. We also thankful to the other medical staffs for helping us in various ways to conduct this study

\section{References:}

Bhandari, M., Devereaux, P.J., Swiontkowski, M.F., Tornetta, P., Obremskey, W., Koval, K.J., Nork, S., Sprague, S., Schemitsch, E.H. \& Guyatt, G.H. (2003): Internal fixation compared with arthroplasty for displaced fractures of the femoral neck. $I$ Bone Joint Surg., 85(9):1673-1681.

Boone, C., Carlberg, K.N., Koueiter, D.M., Baker, K.C., Sadowski, J., Wiater, P.J., Nowinski, G.P. \& Grant, K.D. (2014): Short versus long intramedullary nails for treatment of intertrochanteric femur fractures (OTA31-A1 and $\left.\mathrm{A}_{2}\right)$. L. Orthop. Trauma., 28(5):e96-e10o.

Christodoulou, N.A. \& Sdrenias, C.V. (2000): External fixation of select intertrochanteric fractures with single hip screw. $\underline{\text { Clin. }}$ Orthopaed. Relat. Res., 381:204-211.

Dunn, J., Kusnezov, N., Bader, J., Waterman, B.R., Orr, J. \& Belmont, P.J. (2016): Long versus short cephalomedullary nail for trochanteric femur fractures (OTA $31-\mathrm{A}_{1}, \mathrm{~A}_{2}$ and $\mathrm{A}_{3}$ ): a systematic review.J. Orthopaed. Traumatol.,17(4):361-367.

Soucanye, L.E., Bertani, A., Candoni, P., Charpail, C. \& Demortiere, E.(2012): Proximal femoral nail antirotation (PFN-ATM) fixation of extra-capsular proximal femoral fractures in the elderly: retrospective study in 102 patients, Orthop. Traumatol. Surg. Res., 98(3):288-295.

Rockwood, C.A., Bucholz, R.W., Court-Brown, C.M., Heckman, J.D., Tornetta, P. (2010): Rockwood and Green's fractures in adults, Vol.-1.Pub. by: Lippincott Williams \& Wilkins. P. 2213.

Harandi, B.A., Jafari, S.M. \& Harandi, A.A. (2006): Unstable intertrochanteric hip fractures. Iranian J. Orthpaed.Surg., 4(4):49-57.

Horowitz, B. (1996): Retrospective analysis of hip fractures. Surg. Gynecol. Obstet.,123(3):565-570.

Kanakaris, N.K., Tosounidis, T.H. \& Giannoudis, P.V. (2015): Nailing intertrochanteric hip fractures: short versus long; locked versus nonlocked.J. Orthopaed. Trauma., 29(S4):S1o-6.

Kleweno, C., Morgan, J., Redshaw, J., Harris, M., Rodriguez, E., Zurakowski, D., Vrahas, M. \& Appleton, P. (2014): Short versus long cephalomedullary nails for the treatment of intertrochanteric hip fractures in patients older than 65 years. I. Orthop. Trauma., 28(7):391-397.

Krigbaum, H., Takemoto, S., Kim, H.T. \& Kuo, A.C. (2016): Costs and complications of short versus long cephalomedullary nailing of OTA $31-\mathrm{A} 2$ proximal femur fractures in US veterans. J. Orthop. Trauma., 30(3):125-129.

Loizou, C.L., McNamara, I., Ahmed, K., Pryor, G.A. \& Parker. M.J. (2010): Classification of subtrochanteric femoral fractures. Injury.,41(7):739-745.

Min, W.K., Kim, S.Y., Kim, T.K., Lee, K.B., Cho, M.R., Ha, Y.C. \& Koo, K.H., (2007): Proximal femoral nail for the treatment of reverse obliquity intertrochanteric fractures compared with gamma nail.J. Trauma, 63(5):1054-1060.

Okcu, G., Ozkayin, N., Okta, C., Topcu, I. \& Aktuglu, K. (2013): Which implant is better for treating reverse obliquity fractures 


\section{ORIGINAL ARTICLE}

of the proximal femur: a standard or long nail? Clin. Orthop. Relat. Res., 471(9):2768-2775.

Ramakrishnan, M., Prasad, S.S., Parkinson, R.W. \& Kaye, J.C. (2004): Management of subtrochanteric femoral fractures and metastases using long proximal femoral nail. Injury., 35(2):184190.

Rha, J.D., Kim, Y.H., Yoon, S.I., Park, T.S. \& Lee, M.H., (1993): Factors affecting sliding of the lag screw in intertrochanteric fractures. Int. Orthop., 17(5):320-324.

Schmidt, A.H. \& Swiontkowski, M.F. (2002): Femoral neck fractures. Orthop. Clin. North Am. ,33(1):97-111.
Ambient Science, 2019: Vol. 06h(1); 47-51 DOI:10.21276/ambi.2019.06h.1.oa11

Seyfettinoglu, F., Ersan, O., Kovalak, E., Duygun, F., Ozsar, B. \& Ates. Y. (2010): Fixation of femoral neck fractures with three screws: results and complications. Acta Orthop. Traumatol. Turc., 45(1):6-13.

Vaughn, J., Cohen, E., Vopat, B.G., Kane, P., Abbood, E. \& Born, C. (2015): Complications of short versus long cephalomedullary nail for intertrochanteric femur fractures, minimum 1 year follow-up. Eur. J. Orthop. Surg. Traumatol., 25(4):665670.

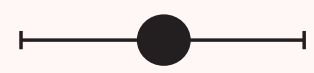

